\title{
Influencia de la microestructura de partida en la ecuación constitutiva para la fluencia de un acero ${ }^{(\cdot)}$
}

\author{
J. Castellanos ${ }^{*}$, V. Gutiérrez ${ }^{*}$ I. Rieiro*, O.A. Ruano** y M. Carsi**
}

Resumen

\begin{abstract}
Se parte de un acero microaleado de grano fino, $20 \mu \mathrm{m}$. Mediante un tratamiento térmico, a este acero se le altera la microestructura, de forma que se consigue un tamaño de grano mucho mayor, $100 \mu \mathrm{m}$. Se realizan ensayos de torsión en probetas con estos dos tamaños de grano a diferentes temperaturas y velocidades de deformación, obteniéndose suficientes datos como para realizar un análisis estadístico fiable. El material de mayor tamaño de grano presenta una tensión de pico mayor que el de tamaño de grano más fino. Este efecto no es debido a una dependencia directa del mecanismo de deformación, ya que la ecuación de Garofalo no depende del tamaño de grano, sino que es debida a la recristalización dinámica que se retarda en los materiales de grano grueso. Además, se dan los resultados de los parámetros de la deformación para cada material. Por último, se aplican las ecuaciones obtenidas a la modelización del conformado de los materiales de grano fino y grueso.
\end{abstract}

\section{Influence of the initial microstructure on the constitutive equation for the creep of a steel}

Abstract

Keywords

\begin{abstract}
The as-started material is a fine grained microalloyed steel, $15-20 \mu \mathrm{m}$. A coarse grain material, $90-100 \mu \mathrm{m}$, is obtained by means of a heat treatment. Torsion tests are conducted in samples with these two grain sizes at various temperatures and strain rates and enough amount of data is obtained to carry out a reliable statistical analysis. The coarser grain material has a higher peak stress than the finer grain material. The constitutive equations are determined for each case and the meaning of the obtained values are discussed. Finally, these equations are applied to the modelling of the forming process of the fine grained and coarse grained materials.
\end{abstract}

Constitutive equations; Microalloyed steel; Creep; Forming simulation.

\section{INTRODUCCIÓN}

La creciente importancia de los modelos de simulación del conformado de materiales hace necesario tanto una mayor fiabilidad de las previsiones como un conocimiento más exacto de los fenómenos físicos involucrados.

Las ecuaciones constitutivas para la fluencia plástica en estado estacionario, en cualquiera de sus marcos definitorios, por ejemplo, los tradicionales de los modelos MATMOD iniciales de Miller y Sherby ${ }^{[1}$ y 2$]$, presentan una serie de condiciones de contorno, entre las que podemos destacar que las soluciones evolucionan en estado estacionario a la ecuación de Garofalo. Uno de los problemas para la obtención de las ecuaciones constitutivas en la modelización de la fluencia plástica es la cantidad de constantes que se deben obtener. La transformación de alguna de estas constantes en una función dependiente de la deformación puede permitir optimizar las ecuaciones constitutivas obteniendo, en lugar de constantes para la caracterización del material, funciones dependientes de la deformación que permitan, además de caracterizar el material, caracterizar la evolución de las condiciones microestructurales con la deformación. Por otra parte, el estado estacionario es difícil de alcanzar en los procesos industriales y tampoco se debe olvidar que las mayores solicitaciones, tanto al material, como a las instalaciones, ocurren cuado la relación tensión deformación alcanza su punto

\footnotetext{
(•) Trabajo recibido el día 18 de septiembre de 2008 y aceptado en su forma final el día 22 de octubre de 2008.

* Dpto. de Matemáticas, Universidad de Castilla-La Mancha, Avda. Carlos III, s/n, 45071 Toledo.

** Dpto. de Metalurgia Física, Centro Nacional de Investigaciones Metalúrgicas (CENIM-CSIC), Avda. Gregorio del Amo 8, 28040 Madrid.
} 


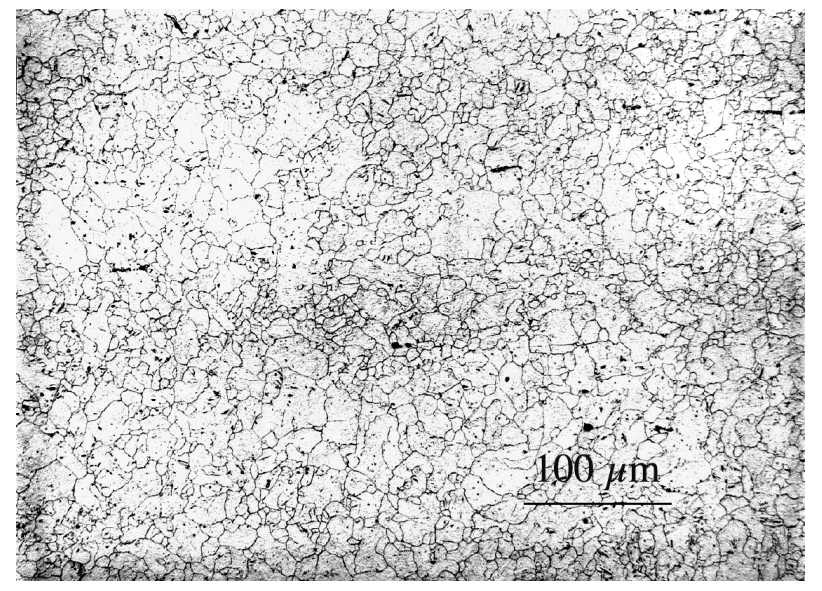

Figura 1. Microestructura de grano fino $(20 \mu \mathrm{m})$.

Figure 1. Fine grain microstructure $(20 \mu \mathrm{m})$.

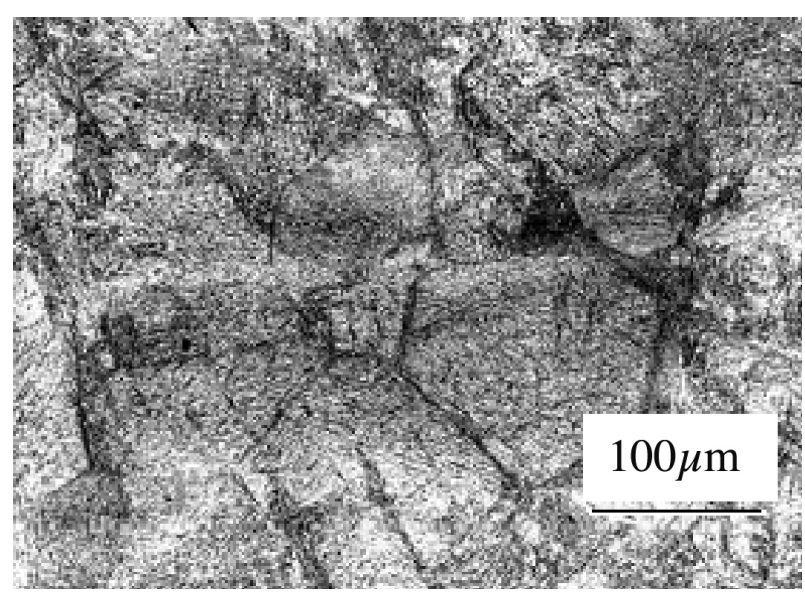

Figura 2. Microstructura de grano grueso $(100 \mu \mathrm{m})$.

\section{Figure 2. Coarse grain microstructure $(100 \mu \mathrm{m})$.}

máximo y, éste, se alcanza con cierta facilidad en procesos de conformado en caliente y semicaliente.

El estudio de la fluencia de los materiales metálicos a temperaturas homologas superiores a 0,6 , considerando, como es bien sabido, temperatura homóloga al cociente de la temperatura de ensayo y la de fusión, distingue, habitualmente, el comportamiento en estado estacionario de otros, no menos interesantes desde el punto de vista de la modelización ${ }^{[3-6]}$ pero cuya ecuación representativa no se puede considerar de estado, sino que depende de las condiciones del material de partida. En particular, el tamaño de grano de la microestructura inicial se ha considerado como determinante en la respuesta de los materiales frente al conformado ${ }^{[7-10]}$. La importancia de cuantificar esta diferencia de comportamiento es obvia: las instalaciones industriales tienen unos límites que no pueden rebasarse, el material al ser conformado es susceptible de agrietarse dependiendo del mapa de estabilidad mecánica ${ }^{[7}$ y 8$]$ y el rendimiento del proceso. Así mismo, los límites de realización del mismo van a venir marcados por los parámetros de la ecuación que va a permitir prever su respuesta ante las solicitaciones exteriores.

La verificación y cuantificación de este hecho, partiendo de un mismo material con dos estructuras de partida, constituye el principal objetivo de este trabajo.

\section{MÉTODO EXPERIMENTAL}

En el presente trabajo se ha utilizado un acero microaleado de composición 0,49 \% C, 0,16\% Mo, $0,19 \% \mathrm{Cu}, 0,12 \% \mathrm{Ni}, 1,06 \% \mathrm{Cr}, 0,12 \% \mathrm{~V},<0,010$ $\% \mathrm{Ti}$, recibido en redondo de $60 \mathrm{~mm}$ de diámetro. Este acero se caracteriza por tener un tamaño de grano bastante fino en términos industriales, habitualmente, $20 \mu \mathrm{m}$. Los microaleantes, entre otras ventajas proporcionan un grano fino y estable frente a tratamientos termomecánicos. Para conseguir una estructura claramente diferenciada se ha tratado parte del acero a $1.220^{\circ} \mathrm{C}$ durante cuatro horas, con lo que se ha obtenido un material de partida con dos tamaños de grano muy diferentes, tal y como se muestra en las figuras 1 y 2 ; en ellas, se puede apreciar que se ha pasado de un tamaño de grano fino $(15-20 \mu \mathrm{m})$ a uno más basto $(100 \mu \mathrm{m})$.

A partir del acero se han preparado 80 probetas de torsión de $6 \mathrm{~mm}$ de diámetro y $17 \mathrm{~mm}$ de longitud útil, para cada estructura de partida, que se han ensayado a rotura en una amplia gama de condiciones para la velocidad generalizada de deformación y temperatura, específicamente entre 850 y $1200^{\circ} \mathrm{C}$ y entre 2 y $24 \mathrm{~s}^{-1}$, de forma que se obtuviera una malla estadísticamente aceptable, El ensayo proporciona directamente el par de torsión frente a número de vueltas. Transformados mediante las ecuaciones de conversión, se obtienen la tensión eficaz y la deformación generalizada. Los tríos de valores obtenidos para la tensión máxima, temperatura y velocidad generalizada de deformación se han analizado mediante un algoritmo desarrollado al efecto y utilizado en otras trabajos anteriores ${ }^{[11 \text { y } 12]}$. También, se ha estudiado la ductilidad del acero para ambas estructuras. La conversión realizada para obtener la conversión a tensión y deformación es la siguiente ${ }^{[8]}$

$$
\begin{gathered}
\sigma=\sqrt{ } 3 \Gamma / 2 \pi \rho^{3}(3+\theta+m) \\
\varepsilon=2 \pi r N / \sqrt{ } 3 L
\end{gathered}
$$


donde, $\Gamma$ es el par, $\mathrm{N}$ es el número de vueltas, $\mathrm{r}$ es el radio, $L$ la longitud eficaz, $\theta$ el coeficiente de endurecimiento y $\mathrm{m}$ la sensibilidad a la velocidad de deformación . Para las probetas utilizadas en este estudio y las características del material, los valores de conversión son $\sigma=32 \Gamma$ y $\varepsilon=0,64 \mathrm{~N}$, es decir, una proporción directa que permite trasladar el análisis de las figuras de una escala a otra.

\section{RESULTADOSY DISCUSIÓN}

En las figuras 3 y 4 se ha comparado el comportamiento en torsión del acero microaleado con dos

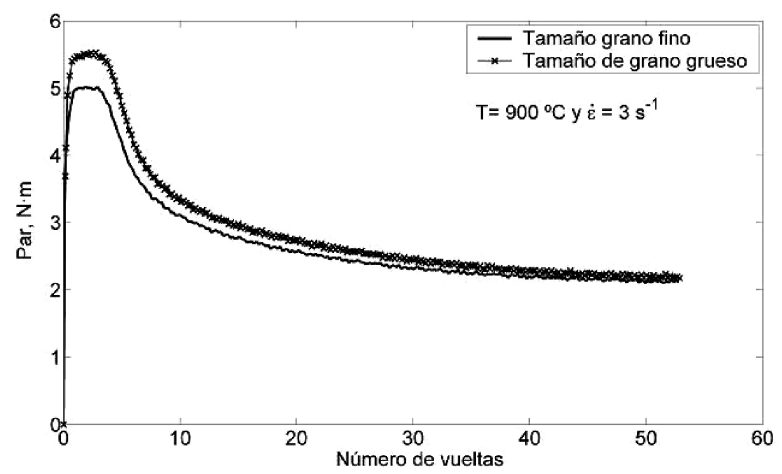

Figura 3. Curvas Par- $\mathrm{N}^{\circ}$ de vueltas a rotura para el acero microaleado con dos tamaños de grano a $900{ }^{\circ} \mathrm{C}$ y $3 \mathrm{~s}^{-1}$.

Figure 3. Curves Torque-Number of turns for the microalloyed steel with two grain sizes at $900{ }^{\circ} \mathrm{C}$ and $3 \mathrm{~s}^{-1}$.

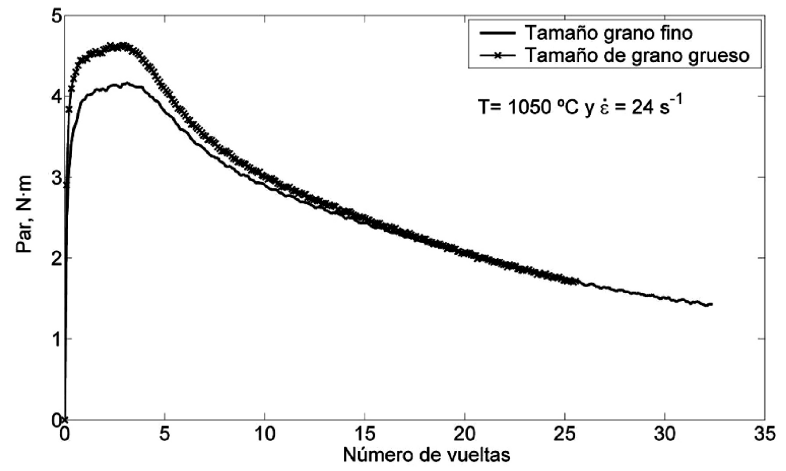

Figura 4. Curvas Par- $N^{\circ}$ de vueltas a rotura para el acero microaleado con dos tamaños de grano a $1050^{\circ} \mathrm{C}$ y $24 \mathrm{~s}^{-1}$.

Figure 4. Curves Torque-Number of turns for the microalloyed steel with two grain sizes of steel at $1050^{\circ} \mathrm{C}$ and $24 \mathrm{~s}^{-1}$. tamaños de grano diferentes, a dos condiciones diferentes de temperatura y velocidad de deformación. Las figuras muestran, sistemáticamente, un valor superior de la tensión (o par) de pico en las probetas con tamaño de grano grueso mientras que el número de vueltas a rotura es similar en ambas microestructuras. Este comportamiento no se puede achacar a una dependencia de la velocidad de deformación con el tamaño de grano ya que la ecuación de Garofalo no depende de éste, sino que se atribuye a la recristalización dinámica que ocurre en este tipo de aceros a las temperaturas de ensayo que se han utilizado en el trabajo, dando lugar a un ablandamiento del material. Es sabido que la recristalización se retarda en los materiales de grano grueso debido a la menor concentración de núcleos de recristalización que se producen, en comparación con los materiales de grano fino. Ello, es debido a que las fronteras de grano son lugares preferentes de nucleación ${ }^{[14}$ y 15]. Por otro lado, al progresar la deformación y, con ella, la recristalización dinámica, las estructuras se unifican ya que, como es bien conocido, el tamaño de grano final, producto de la recristalización dinámica, es independiente del tamaño de grano inicial ${ }^{[16]}$. Por ello, las tensiones en máximo difieren mientras que en estado estacionario deben coincidir.

A partir de los resultados que proporcionan los ensayos de torsión se han calculado las ecuaciones de Garofalo para cada estado inicial mediante un algoritmo desarrollado al efecto ${ }^{[11-13]}$.

Los valores de los parámetros de la ecuación de Garofalo que se han obtenido para ambos tamaños de grano fueron los siguientes:

Grano fino: $Q=278 \mathrm{~kJ} / \mathrm{mol}, \alpha=0,011$

Grano grueso: $\mathrm{Q}=326 \mathrm{~kJ} / \mathrm{mol}, \alpha=0,009$

La bondad de estos parámetros en la predicción del comportamiento de estos materiales se muestra en las figuras 5 y 6 . En ellas, se muestra la representación más corriente de la ecuación de Garofalo

$$
\dot{\varepsilon} \exp Q / R T=\operatorname{Senh}^{n}(\alpha \sigma)
$$

En escala logarítmica se representa, en ordenadas, el primer término, que se conoce como parámetro de Zener-Hollomon y en abscisas, el seno hiperbólico de alfa multiplicado por la tensión. La recta central tiene por pendiente " $n$ " que, a bajas tensiones, puede asimilarse al exponente de la tensión correspondiente a una ley potencial; la banda recta indica los valores con el $95 \%$ de fiabilidad para un error inferior al 5 \%; r es el valor de la correlación y F el coeficiente de Snedecor.

Los valores de las energías de activación para ambos materiales son superiores a los correspondientes 
INFLUENCIA DE LA MICROESTRUCTURA DE PARTIDA EN LA ECUACIÓN CONSTITUTIVA PARA LA FLUENCIA DE UN ACERO INFLUENCE OF THE INITIAL MICROSTRUCTURE ON THE CONSTITUTIVE EQUATION FOR THE CREEP OF A STEEL

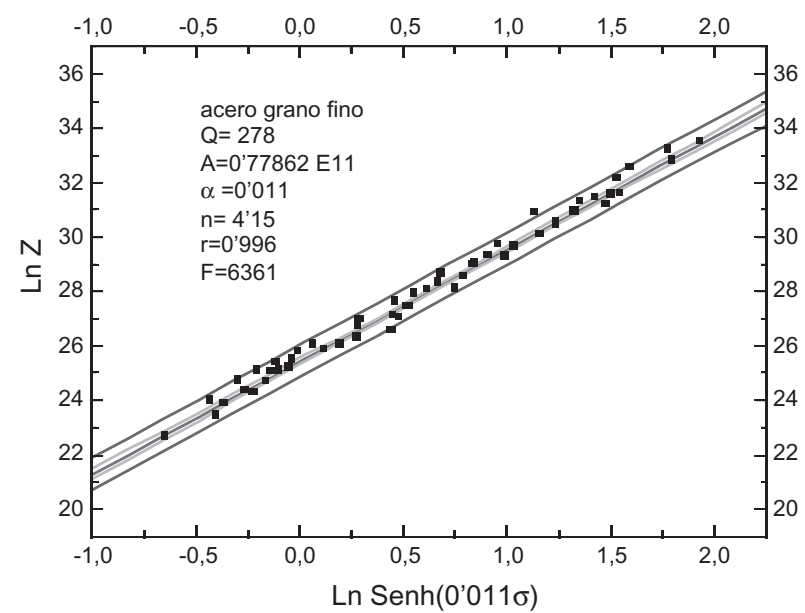

Figura 5. El parámetro de Zener-Hollomon en función de la tensión y el parámetro $\alpha$ para comprobar la bondad de los parámetros de la ecuación de Garofalo para el acero de grano fino.

Figure 5. Zener-Hollomon parameter as a function of stress and $\alpha$ parameter to verify the goodness of the Garofalo equation parameters for the fine grain steel.

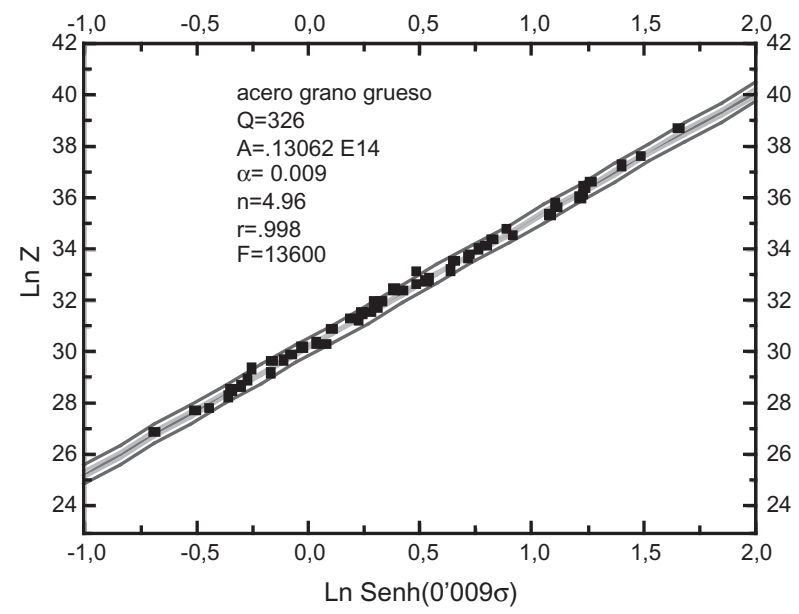

Figura 6. El parámetro de Zener-Hollomon en función de la tensión y el parámetro $\alpha$ para comprobar la bondad de los parámetros de la ecuación de Garofalo para el acero de grano grueso.

Figure 6. Zener-Hollomon parameter as a function of stress and $\alpha$ parameter to verify the goodness of the Garofalo equation parameters for the coarse grain steel.

a la autodifusión de hierro $\gamma(260 \mathrm{~kJ} / \mathrm{mol})$. Esto, es usual cuando se utiliza para el cálculo las tensiones de pico de la curva tensión-deformación. El que sistemáticamente el valor de la tensión en pico sea mayor para el acero de grano grueso no implica que la energía de activación aparente sea superior a la correspondiente al acero de grano fino. Lo que sí implica un aumento en la energía de activación es la mayor diferencia en tensión en el material de grano grueso a igual velocidad de deformación, que es lo que sucede en este caso. Por otro lado, los valores de los parámetros entre ambos tipos de materiales no son muy diferentes. Hay que tener en cuenta que una pequeña variación en uno de los parámetros, especialmente en el alfa, puede tener una influencia en el valor de la energía de activación. De todas maneras, los valores del exponente $\mathrm{n}$ son próximos a 5 , que es el valor correspondiente a una deformación regida por el movimiento de dislocaciones .

Puede parecer sorprendente la gran diferencia existente en los valores de la constante $\mathrm{A}$, que alcanza tres órdenes de magnitud. Sin embargo, hay que considerar que los valores de las constantes de la ecuación de Garofalo solo son comparables a los que poseería la ecuación potencial correspondiente en el dominio de las bajas tensiones, es decir, cuando $\operatorname{senh}(x) \approx x$. Si se comparan los valores de A en estas condiciones se tiene, para el material de grano fino, que:

$$
A_{f}=00^{\prime} 77862 E 11 \times 00^{\prime} 011^{4.15}=579.57
$$

y, para el material de tamaño de grano grueso

$$
A_{g}=0^{\prime} 13062 E 14 \times 00^{\prime} 009^{4.96}=931.27
$$

es decir, magnitudes del mismo orden que, en términos tradicionales, indican una mayor resistencia en el material de grano grueso.

Hay que hacer notar que la energía de activación es menor en estado estacionario, aunque es difícil definir, a altas velocidades de deformación, la deformación correspondiente al mismo. A efectos prácticos, se puede determinar un estado cuasi estacionario en el que las curvas tensión deformación provenientes de las dos estructuras han llegado a converger. La deformación correspondiente es de $\varepsilon=7$, deformación muy superior a aquella en que tienen lugar los fenómenos de recristalización dinámica ${ }^{[6]}$. Se han realizado los cálculos pertinentes para dicha deformación en el material de grano fino obteniéndose $Q=230 \mathrm{~kJ} / \mathrm{mol}$; valor que es, aproximadamente, un $15 \%$ inferior al correspondiente al encontrado en máximo. En el caso de grano grueso la diferencia es mayor, cercana al $20 \%$.

Se han calculado, en cada caso, a partir de las ecuaciones constitutivas del comportamiento en fluencia pertenecientes a los valores de máximo, los mapas de rendimiento y estabilidad mecánica, para 
condiciones de máximo, de acuerdo con un procedimiento basado en las leyes de Liapunov y la potencia gastada en conformar el material ${ }^{[8}$ y 17$]$. Los resultados pueden verse en las figuras 7-10. Las figuras $7 \mathrm{y}$ 8 muestran las zonas de máxima estabilidad mecánica para ambos aceros observándose que para el orden de velocidades de un laminado $\left(20 \mathrm{~s}^{-1}, \ln \varepsilon^{\prime}=3\right)$ se tiene la zona de máxima estabilidad, las de mayor

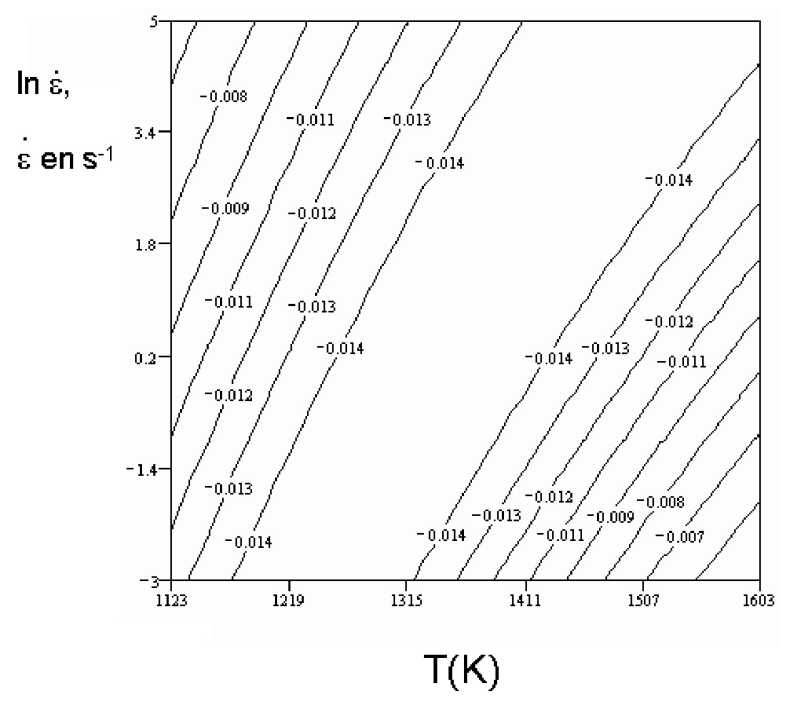

Figura 7. Mapas de estabilidad frente al conformado del acero de grano fino.

Figure 7. Forming stability maps for the fine grain steel.

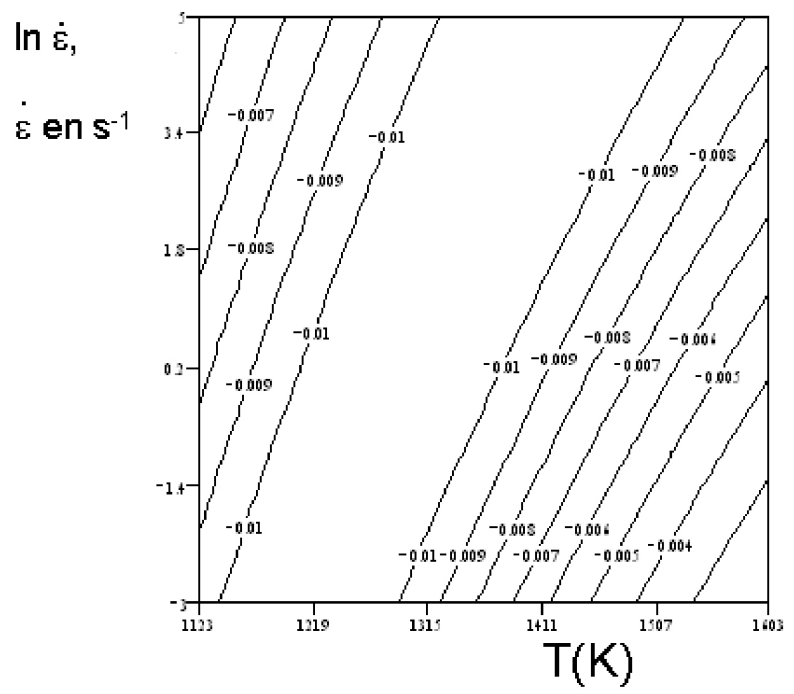

Figura 8. Mapa de estabilidad mecánica del acero de grano grueso.

Figure 8. Forming stability maps for the coarse grain steel.

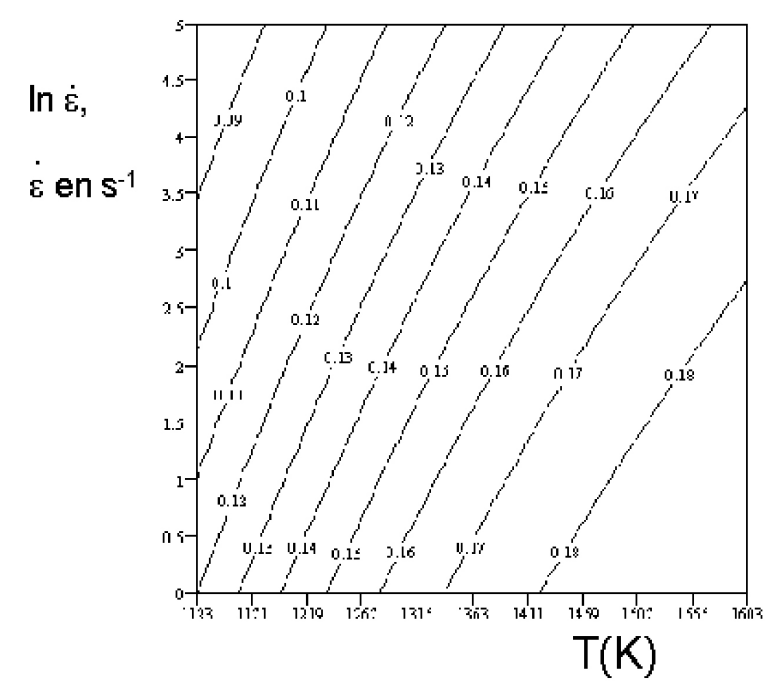

Figura 9. Rendimiento del conformado para el acero de grano fino.

Figure 9. Forming performance of the fine grain steel.

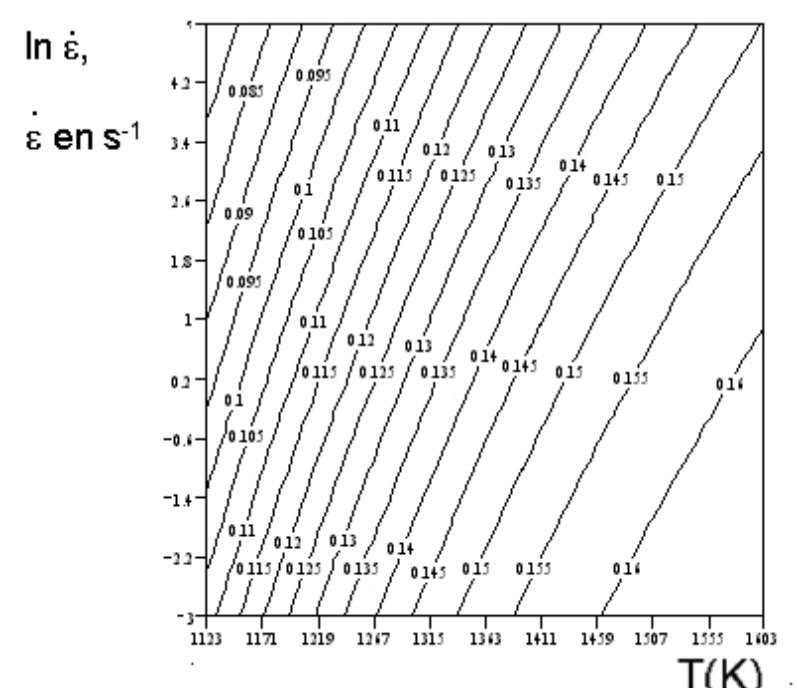

Figura 10. Rendimiento del conformado para el acero de grano grueso.

Figure 10. Forming performance of the coarse grain steel.

valor absoluto, en ambos aceros, a $1.080-1.100{ }^{\circ} \mathrm{C}$. Sin embargo, el acero de grano fino, para la máxima estabilidad mecánica, se encuentra en zona de valores $-0,014$, mientras que el acero de grano grueso está en -0,010, lo que indica que el acero de grano fino tiene una estabilidad $40 \%$ superior al acero de grano grueso frente a grietas de conformado. Por otra parte, en la misma zona de conformado, las figuras 9 
INFLUENCIA DE LA MICROESTRUCTURA DE PARTIDA EN LA ECUACIÓN CONSTITUTIVA PARA LA FLUENCIA DE UN ACERO INFLUENCE OF THE INITIAL MICROSTRUCTURE ON THE CONSTITUTIVE EQUATION FOR THE CREEP OF A STEEL

y 10 proporcionan valores de rendimiento relativo del $15 \%$ para el acero de grano fino y $13 \%$ en el acero de grano grueso, es decir, que la estructura de grano fino, en la mismas condiciones de trabajo, tiene un rendimiento $15 \%$ superior, lo que unido al menor peligro de agrietamiento derivado de los mapas anteriores lo confirman como el que posee una estructura más adecuada para el conformado.

\section{CONCLUSIONES}

- La estructura de partida condiciona la respuesta mecánica de un acero a altas temperaturas.

- Las tensiones en máximo son superiores para el acero de grano grueso. Esto se debe a que la recristalización dinámica se retarda en los materiales de grano grueso.

- Las diferencias en tensiones se atenúan al aumentar la deformación, debido a que la recristalización dinámica iguala, afinando, el tamaño de grano.

- Dado que la ductilidad se mide cuando ya ha ocurrido la recristalización, no hay diferencias sistemáticas según la estructura de partida.

- La energía de activación calculada en máximo es mayor para la estructura de grano grueso.

- El conformado del material de grano fino tiene mejores índices de fiabilidad que el de grano grueso.

\section{Agradecimientos}

Esta trabajo se ha realizado con la ayuda del proyecto PETRI, PET2007-0475-02.

\section{REFERENCIAS}

[1] A.K. Miller y O.D. Sherby, Acta Metall. 26 (1978) 289-304.
[2] A.K. Miller, J. Eng. Mater. Technol. 96 (1976) 97-105.

[3] C. M. Sellars y W.J. McG. Tegart, Mem. Scientif. Rev. Metallurg. LXIII (1966) 7312-746.

[4] F. Garofalo, Trans. AIME 227 (1963) 351-361.

[5] G. R. Canova, S. Shrivastava, J.J. Jonas y C.G. Sellars, Formability of Metallic Materials-2000 A.D. ASTM.,STP-753, 1982, ,J.R.Newby y B.A.Niemeir Eds. American Society for Testing and Materials, pp. 189-210.

[6] I. Rieiro, J. Castellanos, J. Muñoz, F. Peñalba, M. Carsí y O. A. Ruano, Actas XI TRATERMAT, Valencia, España, 2008, pp. 415-418.

[7] M. Carsí, I. Rieiro, J. A. Jiménez, F. Peñalba y O.A. Ruano, J. Mater. Process. Technol. 143. 144 (2003) 416-419.

[8] M. Carsí, R. Allende, F. Peñalba , J.A. Jiménez y O.A. Ruano, Steel Res. Int. 75 (2004) 26-32.

[9] N.D. Ryan y H.J. Mcqueen, J. Mater. Process. Technol. 21 (1990) 177-199.

[10] H. Watanabe, T. Mukai, M. Kohzu, S. Tanabe y K. Higashi, Acta Mater. 47 (1999) 3.753 . 3.758 .

[11] I. Rieiro, M. Carsí y F. Peñalba, Proc. CMT'96, First Int. Conf. Computational Methods and Testing for Engineering Integrity, Kuala Lumpur, Malaysia, 1996, pp 301-312.

[12] I.Rieiro, M.Carsí y F.Peñalba, Rev. Metal. Madrid 32 (1996) 321-328.

[13] I. Rieiro, M. Carsí y O.A. Ruano, Mater.Sci. Technol. (aceptado para publicación en 01/08/2008).

[14] J.J. Jonas, C.M. Sellars y W.J. McG. Tegart, Met. Rev. 4 (1969) 1-23.

[15] H.J. Mcqeen y N.D. Ryan, Mater. Sci. Eng. A 322 (2002) 43-63.

[16] M. Carsi, V. López, F. Peñalba y O.A. Ruano, Mater. Sci. Eng. A 216 (1996) 155-160.

[17] M. Carsí, F. Peñalba, I. Rieiro, F. Zapirain y O.A. Ruano, Z. Metalkde. 91 (2000) 1.0571.062 